\title{
INTRODUCTION: THE NEUROSCIENCE OF READING
}

Lêda M.B. Tomitch

Universidade Federal de Santa Catarina

This special issue of Ilha do Desterro deals with research on the implementation of reading processes in the brain. Basically, the objective of the publication is to put together studies that have investigated the cerebral areas that underlie reading comprehension. The studies reported here involve both typical and atypical subjects, and deal with higher level processes like inferential comprehension, and comprehension monitoring, and also lower level processes like decoding, lexical access, and parsing. Different research methods are used in the seven studies presented here in order to attain the goal of understanding the neural basis of reading: functional Magnetic Resonance Imaging (fMRI), EEG/ERP (Electroencephalogram/ Event Related Potentials), NIRS (Near-Infrared Spectroscopy) and neuropsychological and behavioral measures.

Newman et al's investigation entitled "When syntactic errors go unnoticed: an fMRI study of the effect of semantics on syntax"

\begin{tabular}{|l|l|l|l|l|}
\hline Ilha do Desterro & Florianópolis & $n^{\circ} 63$ & p. 009-014 & jul/dez 2012 \\
\hline
\end{tabular}


used both behavioral and fMRI data to examine the effect of the semantic relatedness of sentence constituents on the ability to detect morphosyntactic (subject/verb agreement) violations. Results from the behavioral data showed that participants were more likely to fail to detect a morphosyntactic violation if the sentence constituents were semantically related to each other than if they were unrelated. It was also found that related anomalous sentences elicited more errors, had a longer reaction and reading times and elicited stronger activation of the left inferior frontal gyrus. Furthermore, three separate clusters of activation were observed including one at BA 44, another at BA 45/46 and a third one at the junction of the inferior frontal and precentral sulci. The authors bring the "good enough" theory of language processing to explain the results; the theory suggests that people fail to generate a complete representation of the input, particularly when the input describes plausible and/or familiar events.

Waldie et al, in a piece of research entitled "Lexical decision making in adults with dyslexia: An Event Related Potential study", examined the timing of neural events with event-related potentials (ERPs) during lexical decision-making between dyslexic readers and unimpaired readers (both unilingual and bilingual). ERPs were calculated for posterior sites in the left and right hemispheres and the P1 and N170 components were compared between groups. Event Related EEG coherence (measuring the synchrony of neural events during lexical tasks both between and within cerebral hemispheres) was also calculated for seven electrode pairs (three pairs at symmetrical locations between hemispheres, and two pairs within each hemisphere). Results showed that individuals with developmental dyslexia have reduced amplitudes in the N170 and higher synchrony between hemispheres during a reading task. The authors explain these differences as possibly being due to 
an asynchrony of neuronal activity at the point where word form features are distinguished.

Baretta et al, in an experimental study entitled "Investigating reading comprehension through EEG", investigated the cerebral processing of both function and content words that were inserted in expository and narrative texts containing suitable or unsuitable conclusions. The N1 component, a negative wave that occurs at approximately $100 \mathrm{msec}$ after stimulus presentation, and seen as a valuable measure of attentional capacity, was used to measure cognitive load while reading the words in the congruent and incongruent paragraphs. Results showed that different scalp areas (midline, right and left hemispheres) responded differently to the influence of the cognitive load in the type of text and word. Lower N1s were found for the content words in the narrative paragraphs and for the function words in the expository texts.

Martin et al, in a study entitled "Influence of reading habits on cerebral plasticity for discourse comprehension in aging", used the Near-Infrared Spectroscopy (NIRS) technique to examine the influence of reading habits on cerebral plasticity in the performance of a comprehension task in aging, which involved reading short stories and answering true or false probes about them. Results showed that the more experienced elderly adults had higher activation in the left superior prefrontal cortex while reading the stories, implying more effort, but had lower activation in this same region when retrieving the information to answer the probe, showing a possible facilitative effect of the initial effort. The authors contend that, cognitive decline in the aging brain, especially that which is related to less efficient short-term and working memory, seems to be compensated by a greater effort to maintain all the information needed to execute a comprehension task. 
Scherer et al, in a study entitled "O processamento da narrativa no envelhecimento e sua relação com memórias de trabalho e episódica e funções executivas", investigated discourse processing in aging by comparing the reading of narratives by young adult readers with that of elderly ones, and also by seeking a relationship between reading ability and episodic and working memory. Results showed that the elderly readers performed worse than the young adult ones in a reading task which measured extratextual coherence including metaphorical and cultural knowledge, and also in relation to the episodic and working memory tests. The elderly group also showed higher response times while answering the comprehension questions and also while performing some of the neuropsychological tests. The authors report evidence indicating a deficit in the executive functions of the elderly readers, when compared to the young adult ones, in relation to the following: ability to concentrate, selectivity of stimuli, ability to abstract, planning, suppression, among others.

Kolinsky et al, in a state-of-the-art article entitled "The impact of orthographic knowledge on speech processing", urge researchers in the field to revise speech recognition models in order to acknowledge the role of reading acquisition. The authors review both behavioral and neuroscientific evidence concerning the role of orthographic knowledge on lexical and post-lexical speech processing, analyzing it with reference to the levels-of-processing approach proposed by Kolinsky (1998) which distinguishes three levels of processing, from lowest to highest: perception, recognition, and formal explicit analysis. Results from research involving illiterate and ex-illiterate Portuguese adults who learned to read and write at adult age are brought to show the impact of orthographic knowledge on metaphonological processing and also to separate alphabetic literacy from the influence of cognitive maturation. An in-depth discussion of whether "orthographic 
knowledge and literacy only influence lexical representations or more basic, perceptual, speech processes" is provided.

Scliar-Cabral, in a state-of-the-art article entitled "Neuroscience applied to learning alphabetic principles: New proposals", reviews recent data on functional illiteracy, discussing them in relation to current advances in the neuroscience of the reading process. The author discusses the psychological reality of invariant features in visual perception of letters, which have been demonstrated by experiments recently run by neuroscientists, focusing on spatial and font invariance, exemplified by a description of invariant features of the Roman alphabet. Two major difficulties faced by beginning readers are also discussed, which include how to dismember the speech chain into words (separated in the written space by blanks) and the syllable into its units, in order to link them to their correspondent graphemes (composed by one or more letters). The author advocates that in order to be able to recognize letters, neurons of the occipito-temporal ventral region of the left hemisphere must be recycled for perceiving the minimal feature differences among them, and this learning will only succeed, if one or more letters, the graphemes, are introduced inside words, where they have the function of distinguishing meaning. The author brings two wellsucceeded intervention initiatives to prevent functional illiteracy.

Besides the research articles just presented, this issue brings two book reviews on the most important publications on the neuroscience of language in the past few years: one specifically about reading- Reading in the Brain- published in 2010, by one of the most renowned contemporary neuroscientists- Stanislas Dehaene; and another which is more general but brings various chapters on reading- Handbook of the Neuroscience of Language- 
published in 2008, by Brigitte Stemmer and Harry Whitaker. The former is reviewed by Almeida and Winfield and the latter by Bailer. The high-quality studies in this issue of Ilha do Desterro report original and relevant research in the area of reading comprehension, elucidating important matters regarding the understanding of the neural basis of language processing, being of interest to both graduate students and scholars from the fields of linguistics, psychology, education, speech therapy and all those interested in the neuroscience of reading. 